\title{
One-Parameter Equations of Spherical Conics and Its Applications
}

\author{
Bülent Altunkaya ${ }^{1}$, Yusuf Yaylı ${ }^{2}$, H. Hilmi Hacısalihoğlu ${ }^{3} \&$ Fahrettin Arslan $^{2}$ \\ ${ }^{1}$ Department of Mathematics, Faculty of Education, University of Ahi Evran, Kırşehir, Turkey \\ 2 Department of Mathematics, Faculty of Sciences, University of Ankara, Ankara, Turkey \\ ${ }^{3}$ Department of Mathematics, Faculty of Sciences, University of Bilecik, Bilecik, Turkey \\ Correspondence: Bülent Altunkaya, Department of Mathematics, Faculty of Education, University of Ahi Evran, \\ Kırşehir, Turkey. E-mail: bulent.altunkaya@ahievran.edu.tr
}

Received: July 22, 2014 Accepted: September 17, 2014 Online Published: October 20, 2014

doi:10.5539/jmr.v6n4p77 URL: http://dx.doi.org/10.5539/jmr.v6n4p77

\begin{abstract}
If we transform definitions of the conics in Euclidean plane on sphere, we obtain spherical conics. To calculate the E. Study Map of the spherical conics, we have to find one parameter equations of them. We had done this before in (Altunkaya, Yaylı, Hacısalihoğlu, \& Arslan, 2011). In this paper, we not only developed the results that we have found before, but also calculated the E. Study Map of the spherical conics when they are great circles by using the theorems in (Hacisalihoğlu, 1977).
\end{abstract}

Keywords: spherical ellipse, spherical hyperbola, spherical parabola, E. study map

\section{Introduction}

W. K. Clifford (1849-79) introduced dual numbers while he was working on developing Clifford algebras. After him, E. Study developed dual vectors and a mapping which is known as E. Study Map. He noticed that, the oriented lines in Euclidean 3-space $E^{3}$ are in one-to-one correspondence with the points of the dual unit sphere $S_{D}^{2}$ (Study, 1903). By the help of this, the geometry of ruled surfaces are represented. For this, you need to have a differentiable curve which depends on a real parameter.

A differentiable curve $X(t)$ on the dual unit sphere $S_{D}^{2}$, depending on a real parameter $t$, represents a differentiable family of straight lines in $E^{3}$ which called as a ruled surface (Guggenheimer, 1977; Veldkamp, 1976).

In (Hacısalihoğlu, 1977), he showed that the E. Study map of a circle on a dual unit sphere $S_{D}^{2}$ is a family of hyperboloids of one sheet with two parameters. In (Altunkaya, Yaylı, Hacisalihoğlu, \& Arslan, 2011) we have found the one parameter equations of the spherical conics, by this we realised that the E. Study Map of the spherical conics can be found.

In this paper, we calculate and discuss the E. Study maps of spherical conics on dual unit sphere $S_{D}^{2}$ when they are great circles, each of which is a geometrical result.

\section{Basic Concepts}

\subsection{Spherical Conics}

Take two fixed points $F_{1}$ and $F_{2}$ on Euclidean plane with $d\left(F_{1}, F_{2}\right)=2 c, 0<c \in R^{+}$, then the locus of the points such that

$$
d\left(X, F_{1}\right)+d\left(X, F_{2}\right)=2 a, \quad 0<c<a \in R^{+}, \quad X \in R^{2}
$$

is called a planar ellipse.

Given two points $P$ and $Q$ on unit sphere $S^{2} \subset R^{3}$, let $\theta$ be the angle subtended at the center of the unit sphere by $P Q$ and $d(P, Q)$ denote the geodesic distance between $P$ and $Q$. If we apply planar ellipse definition on $S^{2}$ then we may define spherical ellipse. We will define spherical hyperbola and spherical parabola similarly. 


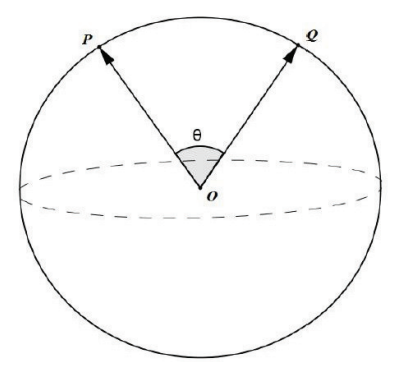

Figure 1. Geodesic distance

Definition 1 Take two fixed points $F_{1}$ and $F_{2}$ on $S^{2}$ with the properties of $d\left(F_{1}, F_{2}\right)=2 c, 0<c<a \in R^{+}$, the set of points

$$
S=\left\{X \mid \quad d\left(X, F_{1}\right)+d\left(X, F_{2}\right)=2 a, \quad X \in S^{2}\right\}
$$

is called spherical ellipse (Figure 2).

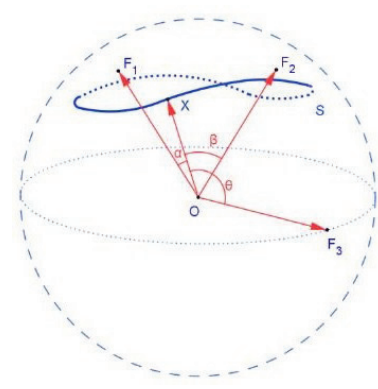

Figure 2. Spherical ellipse

From now on, we will use $\overrightarrow{F_{1}}, \overrightarrow{F_{2}}$, and $\overrightarrow{F_{3}}$ for the position vectors of the points $F_{1}, F_{2}$, and $F_{3}$, respectively.

If we take $\overrightarrow{F_{3}}=\frac{\overrightarrow{F_{1}} \times \overrightarrow{F_{2}}}{\left\|\overrightarrow{F_{1}} \times \overrightarrow{F_{2}}\right\|}$, then $\overrightarrow{F_{1}}, \overrightarrow{F_{2}}$ and $\overrightarrow{F_{3}}$ will be linearly independent. Hence, for any point $X$ on $S$ we can write

$$
\vec{X}=\frac{\cos t-\cos (2 a-t) \cos 2 c}{\sin ^{2} 2 c} \overrightarrow{F_{1}}+\frac{\cos (2 a-t)-\cos t \cos 2 c}{\sin ^{2} 2 c} \overrightarrow{F_{2}}+\cos \theta \overrightarrow{F_{3}}
$$

while

$$
X=\left(x_{1}, x_{2}, x_{3}\right),\|\vec{X}\|^{2}=1, X \in S^{2}
$$

After combining (1) with (2) we can have $X$ depending on one parameter (Altunkaya, Yaylı, Hacisalihoğlu, \& Arslan, 2011).

Definition 2 Take two fixed points $F_{1}$ and $F_{2}$ on $S^{2}$ with the properties of $d\left(F_{1}, F_{2}\right)=2 c, 0<c \in R^{+}$, the set of points

$$
S=\left\{X|\quad| d\left(X, F_{1}\right)-d\left(X, F_{2}\right) \mid=2 a, \quad a \in R^{+} \cup\{0\}, X \in S^{2}\right\}
$$

is called spherical hyperbola.

If we take $\overrightarrow{F_{3}}=\frac{\overrightarrow{F_{1}} \times \overrightarrow{F_{2}}}{\left\|\vec{F}_{1} \times \vec{F}_{2}\right\|}$, then $\overrightarrow{F_{1}}, \overrightarrow{F_{2}}$ and $\overrightarrow{F_{3}}$ will be linearly independent. Hence, for any point $X$ on $S$, we can write two equations if $t>\beta$

$$
\vec{X}=\frac{\cos t-\cos (t-2 a) \cos 2 c}{\sin ^{2} 2 c} \overrightarrow{F_{1}}+\frac{\cos (t-2 a)-\cos t \cos 2 c}{\sin ^{2} 2 c} \overrightarrow{F_{2}}+\cos \theta \overrightarrow{F_{3}}
$$

and if $t<\beta$

$$
\vec{X}=\frac{\cos t-\cos (t+2 a) \cos 2 c}{\sin ^{2} 2 c} \overrightarrow{F_{1}}+\frac{\cos (t+2 a)-\cos t \cos 2 c}{\sin ^{2} 2 c} \overrightarrow{F_{2}}+\cos \theta \overrightarrow{F_{3}}
$$


After combining (3) with (2) and (4) with (2), we can have $X$ depending on only one parameter (Altunkaya, Yayl, Hacısalihoğlu, \& Arslan, 2011).

As we know, the definition of the planar parabola is the locus of points in that plane that are equidistant from both the directrix(line) and the focus(point). If we transform the definition of the planar parabola on sphere, we have to use great circles instead of directrix(line). Now, we can give a new definition.

Definition 3 Take two fixed points $F_{1}$ and $F_{2}$ on $S^{2}$ with the properties of $d\left(F_{1}, F_{2}\right)=2 c, 0<c \in R^{+}$and $S^{\prime}$ be the great circle whose normal vector is $\overrightarrow{F_{2}}$, the set of points

$$
S=\left\{X \mid \quad d\left(X, F_{1}\right)+\frac{\pi}{2}=d\left(X, F_{2}\right), \quad X \in S^{2}\right\}
$$

is called spherical parabola.

If we take $\overrightarrow{F_{3}}=\frac{\overrightarrow{F_{1}} \times \overrightarrow{F_{2}}}{\left\|\overrightarrow{F_{1}} \times \overrightarrow{F_{2}}\right\|}$, then $\overrightarrow{F_{1}}, \overrightarrow{F_{2}}$ and $\overrightarrow{F_{3}}$ will be linearly independent. Hence, for any point $X$ on $S$, we can write

$$
\vec{X}=\frac{\cos t-\sin t \cos 2 c}{\sin ^{2} 2 c} \overrightarrow{F_{1}}+\frac{-\sin t-\cos t \cos 2 c}{\sin ^{2} 2 c} \overrightarrow{F_{2}}+\cos \theta \overrightarrow{F_{3}}
$$

After combining (5) with (2), we can have $X$ depending on one parameter (Altunkaya, Yaylı, Hacısalihoğlu, \& Arslan, 2011).

Example 1 Let us take $\overrightarrow{F_{1}}=(1,0,0)$ and $\overrightarrow{F_{2}}=(0,1,0)$, then we can write $\overrightarrow{F_{3}}=\frac{\overrightarrow{F_{1}} \times \overrightarrow{F_{2}}}{\left\|\overrightarrow{F_{1}} \times \overrightarrow{F_{2}}\right\|}=(0,0,1)$. If we plug in $\overrightarrow{F_{1}}, \overrightarrow{F_{2}}, \overrightarrow{F_{3}}$ and $2 a=\frac{2 \pi}{3}$ in Equation (1) then combine it with (2), we will have the following equations

$$
\begin{gathered}
x_{1}=\cos t \\
x_{2}=\cos \left(\frac{2 \pi}{3}-t\right) \\
x_{3}= \pm \sqrt{1-\cos ^{2} t-\cos ^{2}\left(\frac{2 \pi}{3}-t\right)}
\end{gathered}
$$

In these three equations, for $t \in\left[\frac{\pi}{12}, \frac{7 \pi}{12}\right]$ we will have the following graphs.
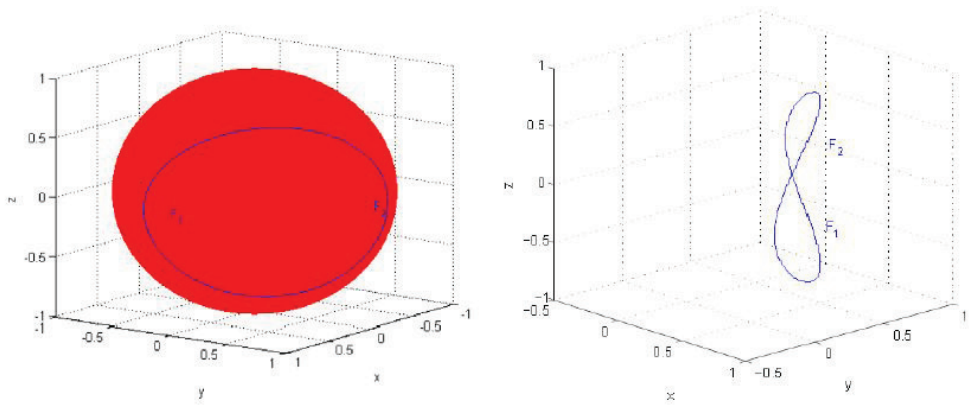

Figure 3. Spherical ellipse with and without sphere

We have this graph with the Matlab command below

\section{holdon;}

ezplot $3\left({ }^{\prime} \cos (t)^{\prime},{ }^{\prime} \cos (2 * p i / 3-t)^{\prime},{ }^{\prime} \operatorname{sqr} t\left(1-(\cos (t)) .^{2}-(\cos (2 * p i / 3-t)) .^{2}\right)^{\prime}\right.$, $[\mathrm{pi} / 12,(7 * \mathrm{pi}) / 12])$,

ezplot3 $\left({ }^{\prime} \cos (t)^{\prime},{ }^{\prime} \cos (2 * p i / 3-t)^{\prime},{ }^{\prime}-\operatorname{sqrt}\left(1-(\cos (t)) .^{2}-(\cos (2 * p i / 3-t)) .^{2}\right)^{\prime}\right.$, $[p i / 12,(7 * p i) / 12])$,

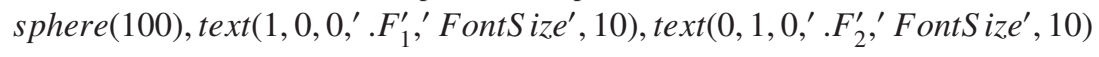




\subsection{Dual Numbers}

A dual number $\tilde{u}$ is an ordered pair $\left(u, u^{*}\right)$ for all $u, u^{*} \in R$. If we denote dual number set with $D$, dual numbers can be written as,

$$
D=\left\{\left(u, u^{*}\right) \mid u, u^{*} \in R\right\}
$$

Two inner eqations and the equality on $D$ are defined as follows (Körpınar \& Turhan, 2010):

For all $\tilde{u}=\left(u, u^{*}\right), \tilde{v}=\left(v, v^{*}\right) \in D$,

$$
\begin{gathered}
\tilde{u}+\tilde{v}=\left(u, u^{*}\right)+\left(v, v^{*}\right)=\left(u+v, u^{*}+v^{*}\right) \\
\tilde{u} \tilde{v}=\left(u, u^{*}\right)\left(v, v^{*}\right)=\left(u v, u v^{*}+u^{*} v\right) \\
\tilde{u}=\tilde{v} \Longleftrightarrow u=v \quad \wedge \quad u^{*}=v^{*} .
\end{gathered}
$$

We can easily see the set of dual numbers is a commutative ring. $e=(1,0)$ is called the unit element of multiplication and $\varepsilon=(0,1)$ is called the dual unit.

The function

$$
\begin{aligned}
& f: D \rightarrow R \\
& (u, 0) \rightarrow u
\end{aligned}
$$

is an isometry (Veldkamp, 1976).

So, we can take $(a, 0)$ as $a$. If we make the calculations we can easily see,

$$
\varepsilon^{2}=\varepsilon \varepsilon=(0,0)=0
$$

and

$$
\tilde{u}=\left(u, u^{*}\right)=(u, 0)+(0,1)\left(u^{*}, 0\right)=u+\varepsilon u^{*} .
$$

Clifford showed that dual numbers form an algebra, not a field. The pure dual numbers $\varepsilon u^{*}$ are zero divisors, $\left(\varepsilon u^{*}\right)\left(\varepsilon v^{*}\right)=0$. However, the other laws of the algebra of dual numbers are the same as the laws of algebra of complex numbers. This means dual numbers form a ring over the real number field. Now, let $f$ be a differentiable dual function. Thus Taylor expansion of dual function $f$ is

$$
f\left(x+\varepsilon x^{*}\right)=f(x)+\varepsilon x^{*} f^{\prime}(x)
$$

where $f^{\prime}(x)$ is the derivation of $f$, then we have

$$
\begin{aligned}
& \sin \left(x+\varepsilon x^{*}\right)=\sin x+\varepsilon x^{*} \cos x \\
& \cos \left(x+\varepsilon x^{*}\right)=\cos x-\varepsilon x^{*} \sin x
\end{aligned}
$$

Now we can talk about dual vectors. The set

$$
D^{3}=\left\{\tilde{u}=\left(\tilde{u_{1}}, \tilde{u_{2}}, \tilde{u_{3}}\right) \mid \tilde{u}_{i} \in D, 1 \leq i \leq 3\right\}
$$

is a module over the ring $D$ which is called $D$-Module or dual space. The elements of $D^{3}$ are called dual vectors.

Thus, a dual vector $\overrightarrow{\tilde{u}}$ can be written as,

$$
\overrightarrow{\tilde{u}}=\vec{u}+\varepsilon \overrightarrow{u^{*}}
$$

where $u$ and $u^{*}$ are real vectors in $E^{3}$. Then for any vectors $\overrightarrow{\vec{u}}$ and $\vec{v}$ in $D^{3}$, the scalar product and the vector product are defined by (Veldkamp, 1976)

$$
\langle\overrightarrow{\tilde{u}}, \overrightarrow{\widetilde{v}}\rangle=\langle\vec{u}, \vec{v}\rangle+\varepsilon\left(\left\langle\vec{u}, \overrightarrow{v^{*}}\right\rangle+\left\langle\overrightarrow{u^{*}}, \vec{v}\right\rangle\right)
$$

and

$$
\overrightarrow{\tilde{u}} \wedge \overrightarrow{\tilde{v}}=\vec{u} \wedge \vec{v}+\varepsilon\left(\vec{u} \wedge \overrightarrow{v^{*}}+\overrightarrow{u^{*}} \wedge \vec{v}\right)
$$


The norm of a dual vector $\overrightarrow{\tilde{u}}$ is defined to be (Veldkamp, 1976)

$$
\|\overrightarrow{\tilde{u}}\|=\|\vec{u}\|+\varepsilon \frac{\left\langle\vec{u}, \overrightarrow{u^{*}}\right\rangle}{\|\vec{u}\|} \in D, \quad \vec{u} \neq 0 .
$$

A dual vector $\overrightarrow{\tilde{u}}$ with $\|\overrightarrow{\tilde{u}}\|=1$ is called dual unit vector and the set

$$
S_{D}^{2}=\left\{\overrightarrow{\tilde{u}}=\vec{u}+\varepsilon \overrightarrow{u^{*}} \mid \quad\|\overrightarrow{\tilde{u}}\|=(1,0), \quad \vec{u}, \overrightarrow{u^{*}} \in E^{3}\right\}
$$

is called dual unit sphere with the center $\tilde{0}$ in $D^{3}$.

E. Study gave the definition of a mapping

$$
D-\text { Module } \longrightarrow R^{3}
$$

named as Study Mapping and the following theorem.

Theorem 1 (Study, 1903) The oriented lines in $E^{3}$ are in one to one correspondence between the points of dual unit sphere, in D - Module.

With the help of Theorem 1, we can see a dual point on $S_{D}^{2}$ corresponds to a line in $E^{3}$.

E. Study introduced the dual angle subtended by two nonparallel lines in $E^{3}$ and defined it as $\tilde{\varphi}=\varphi+\varepsilon \varphi^{*} \in D$.

Let $X, Y$ denote two different points of $S_{D}^{2}$ and $\tilde{\varphi}=\varphi+\varepsilon \varphi^{*}$ be the dual angle between these two lines. E.Study showed that, $\varphi$ is the projected angle and $\varphi^{*}$ is the shortest distance between the corresponding lines in $E^{3}$.

Theorem 2 (Hacisalihoğlu, 1977) $\langle\vec{X}, \vec{Y}\rangle=\cos \tilde{\varphi}=\cos \varphi-\varepsilon \varphi^{*} \sin \varphi, \quad \tilde{\varphi}=\varphi+\varepsilon \varphi^{*} \in D, \quad \forall \vec{X}, \vec{Y} \in D^{3}$.

The following special cases of Theorem 2 are important (Hacisalihoğlu, 1977).

$$
\langle\vec{X}, \vec{Y}\rangle=0 \Longrightarrow \varphi=\frac{\pi}{2}, \quad \varphi^{*}=0
$$

this means that the lines $\vec{X}$ and $\vec{Y}$ meet at a right angle.

$$
\langle\vec{X}, \vec{Y}\rangle=\text { pure } \quad \text { dual } \Longrightarrow \varphi=\frac{\pi}{2}, \quad \varphi^{*} \neq 0 ;
$$

this means that the lines $\vec{X}$ and $\vec{Y}$ are orthogonal skew lines.

$$
\langle\vec{X}, \vec{Y}\rangle=\text { pure } \quad \text { real } \Longrightarrow \varphi \neq \frac{\pi}{2}, \quad \varphi^{*}=0 ;
$$

this means that the lines $\vec{X}$ and $\vec{Y}$ intersect each other.

$$
\langle\vec{X}, \vec{Y}\rangle= \pm 1 \Longrightarrow \varphi=0, \quad \varphi^{*}=0
$$

this means that the lines $\vec{X}$ and $\vec{Y}$ are coincident.

If we take a differentiable curve on $S_{D}^{2}$, depending on a real parameter $t$, represents a differentiable family of straight lines of $R^{3}$ which is a ruled surface. The lines $\overrightarrow{X(t)}$ are the generators of the surface.

Theorem 3 (Hacisalihoğlu, 1977) Let $S$ be a circle on $S_{D}^{2}$, then the E. Study Map of $S$ is a family of hyperboloid of one sheet with two parameters.

Definition 4 (Hacısalihoğlu, 1977) If all the lines of a line congruence orthogonally intersect a constant line then the congruence is called a recticongruence.

Theorem 4 Let $S$ be a great circle on $S_{D}^{2}$, that is

$$
S=\left\{\vec{X} \mid\langle\vec{X}, \vec{G}\rangle=0, \quad \vec{X}, \vec{G} \in S_{D}^{2}\right\}
$$


Then the Study map of $S$ is a recticongruence.

3 E. Study map of the spherical conics when they are great circles

Theorem 5 Let $X(t): I \longrightarrow S^{2}$ be a spherical ellipse defined at Definition 1 with the foci $F_{1}$ and $F_{2} \in S^{2}$. Then, $X(t)$ is a circle if $F_{1}= \pm F_{2}$ or $2 a=\pi$.

Proof. If $F_{1}= \pm F_{2}$ it is obvious that $X$ is a circle. If $2 a=\pi$ and $F_{1} \neq F_{2}$ then

$$
\begin{aligned}
\overrightarrow{X(t)} & =\frac{\cos t-\cos (2 a-t) \cos 2 c}{\sin ^{2} 2 c} \overrightarrow{F_{1}}+\frac{\cos (2 a-t)-\cos t \cos 2 c}{\sin ^{2} 2 c} \overrightarrow{F_{2}}+\cos \theta \overrightarrow{F_{3}} \\
& =\frac{\cos t+\cos t \cos 2 c}{\sin ^{2} 2 c} \overrightarrow{F_{1}}+\frac{-\cos t-\cos t \cos 2 c}{\sin ^{2} 2 c} \overrightarrow{F_{2}}+\cos \theta \overrightarrow{F_{3}} \\
& =\frac{\cos t(1+\cos 2 c)}{\sin ^{2} 2 c} \overrightarrow{F_{1}}-\frac{\cos t(1+\cos 2 c)}{\sin ^{2} 2 c} \overrightarrow{F_{2}}+\cos \theta \overrightarrow{F_{3}} \\
& =\frac{\cos t\left(1+2 \cos ^{2} c-1\right)}{\sin ^{2} 2 c} \overrightarrow{F_{1}}-\frac{\cos t\left(1+2 \cos ^{2} c-1\right)}{\sin ^{2} 2 c} \overrightarrow{F_{2}}+\cos \theta \overrightarrow{F_{3}} \\
& =\frac{2 \cos t \cos ^{2} c}{4 \sin ^{2} c \cos ^{2} c} \overrightarrow{F_{1}}-\frac{2 \cos t \cos ^{2} c}{4 \sin ^{2} c \cos ^{2} c} \overrightarrow{F_{2}}+\cos \theta \overrightarrow{F_{3}} \\
& =\frac{\cos t}{2 \sin ^{2} c} \overrightarrow{F_{1}}-\frac{\cos t}{2 \sin ^{2} c} \vec{F}_{2}+\cos \theta \overrightarrow{F_{3}}
\end{aligned}
$$

The inner product

$$
\begin{aligned}
\overrightarrow{X(t)} \cdot\left(\overrightarrow{F_{1}}+\overrightarrow{F_{2}}\right) & =\left(\frac{\cos t}{2 \sin ^{2} c} \overrightarrow{F_{1}}-\frac{\cos t}{2 \sin ^{2} c} \overrightarrow{F_{2}}+\cos \theta \overrightarrow{F_{3}}\right) \cdot\left(\overrightarrow{F_{1}}+\overrightarrow{F_{2}}\right) \\
& =0
\end{aligned}
$$

shows that the set

$$
S=\left\{X \mid \quad d\left(X, F_{1}\right)+d\left(X, F_{2}\right)=\pi, \quad X \in S^{2}\right\}
$$

is a great circle whose normal is $\overrightarrow{F_{1}}+\overrightarrow{F_{2}}$.

Theorem 6 Let $X(t): I \longrightarrow S^{2}$ be a spherical hyperbola defined at Definition 2 with the foci $F_{1}$ and $F_{2} \in S^{2}$. Then, $X(t)$ is a circle if $F_{1}= \pm F_{2}$ or $2 a=0$.

Proof. If $F_{1}= \pm F_{2}$ it is obvious that $X$ is a circle. If $2 a=0$ and $F_{1} \neq F_{2}$, then

$$
\begin{aligned}
\overrightarrow{X(t)} & =\frac{\cos t-\cos (t \pm 2 a) \cos 2 c}{\sin ^{2} 2 c} \overrightarrow{F_{1}}+\frac{\cos (t \pm 2 a)-\cos t \cos 2 c}{\sin ^{2} 2 c} \overrightarrow{F_{2}}+\cos \theta \overrightarrow{F_{3}} \\
& =\frac{\cos t-\cos t \cos 2 c}{\sin ^{2} 2 c} \overrightarrow{F_{1}}+\frac{\cos t-\cos t \cos 2 c}{\sin ^{2} 2 c} \overrightarrow{F_{2}}+\cos \theta \overrightarrow{F_{3}} \\
& =\frac{\cos t(1-\cos 2 c)}{\sin ^{2} 2 c} \overrightarrow{F_{1}}+\frac{\cos t(1-\cos 2 c)}{\sin ^{2} 2 c} \overrightarrow{F_{2}}+\cos \theta \overrightarrow{F_{3}} \\
& =\frac{\cos t\left(1+2 \sin ^{2} c-1\right)}{\sin ^{2} 2 c} \overrightarrow{F_{1}}+\frac{\cos t\left(1+2 \sin ^{2} c-1\right)}{\sin ^{2} 2 c} \overrightarrow{F_{2}}+\cos \theta \overrightarrow{F_{3}} \\
& =\frac{2 \cos t \sin ^{2} c}{4 \sin ^{2} c \cos ^{2} c} \overrightarrow{F_{1}}+\frac{2 \cos t \sin ^{2} c}{4 \sin ^{2} c \cos ^{2} c} \overrightarrow{F_{2}}+\cos \theta \overrightarrow{F_{3}} \\
& =\frac{\cos t}{2 \cos ^{2} c} \overrightarrow{F_{1}}+\frac{\cos t}{2 \cos ^{2} c} \vec{F}_{2}+\cos \theta \overrightarrow{F_{3}}
\end{aligned}
$$

The inner product

$$
\begin{aligned}
\overrightarrow{X(t)} \cdot\left(\overrightarrow{F_{1}}-\overrightarrow{F_{2}}\right) & =\left(\frac{\cos t}{2 \cos ^{2} c} \overrightarrow{F_{1}}+\frac{\cos t}{2 \cos ^{2} c} \overrightarrow{F_{2}}+\cos \theta \overrightarrow{F_{3}}\right) \cdot\left(\overrightarrow{F_{1}}-\overrightarrow{F_{2}}\right) \\
& =0
\end{aligned}
$$

shows that the set

$$
S=\left\{X|\quad| d\left(X, F_{1}\right)-d\left(X, F_{2}\right) \mid=0, \quad X \in S^{2}\right\}
$$


is a great circle whose normal is $\overrightarrow{F_{1}}-\overrightarrow{F_{2}}$.

Theorem 7 Let $X(t): I \longrightarrow S^{2}$ be a spherical parabola defined at Definition 3 with $F_{1}$ and $F_{2} \in S^{2}$. Then, $X(t)$ is a circle if $d\left(F_{1}, F_{2}\right)=\frac{\pi}{2}$.

Proof. If in (5) we take $d\left(F_{1}, F_{2}\right)=\frac{\pi}{2}$, then

$$
\begin{aligned}
\overrightarrow{X(t)} & =\frac{\cos t+\sin t \cos 2 c}{\sin ^{2} 2 c} \overrightarrow{F_{1}}+\frac{-\sin t-\cos t \cos 2 c}{\sin ^{2} 2 c} \overrightarrow{F_{2}}+\cos \theta \overrightarrow{F_{3}} \\
& =\frac{\cos t+\sin t \cdot 0}{1} \overrightarrow{F_{1}}+\frac{-\sin t-\cos t \cdot 0}{1} \overrightarrow{F_{2}}+\cos \theta \overrightarrow{F_{3}} \\
& =\cos t \overrightarrow{F_{1}}-\sin t \overrightarrow{F_{2}}+\cos \theta \overrightarrow{F_{3}}
\end{aligned}
$$

The inner product

$$
\overrightarrow{X(t)} \cdot \overrightarrow{F_{3}}=\cos \theta
$$

While $\overrightarrow{F_{2}}$ is the normal vector of $S^{\prime}$ and $d\left(F_{1}, F_{2}\right)=\frac{\pi}{2}$, then $\overrightarrow{F_{1}}$ is a point on $S^{\prime}$. So, $\theta=\frac{\pi}{2}$, then

$$
\overrightarrow{X(t)} \cdot \overrightarrow{F_{3}}=\cos \frac{\pi}{2}=0
$$

shows that the set

$$
S=\left\{X \mid \quad d\left(X, F_{1}\right)+\frac{\pi}{2}=d\left(X, F_{2}\right), \quad X \in S^{2}\right\}
$$

is a great circle whose normal is $\overrightarrow{F_{3}}$.

As we see the spherical conics can be great circles. So, from Theorem 3 and Theorem 4 we can say that the E. Study map of spherical conics can be a recticongruence and a hyperboloid of one sheet.

\section{Conclusion}

Since we can summarize ruled surfaces as the set of points which are swept by a moving line, this kind of surfaces has a wide range of application such as Computer Aided Geometric Design(CAGD), surface fitting and interpolating in computational and differential geomety, simulation of rigid bodies of robots and spatial mechanisms, 3D object recognition and reconstruction, designing flank surface of fan, compressor and impeller blades (Wu, 1995), the dual inverse mapping and optimal generation of ruled surfaces, moving geometry and kinematics for modeling problems.

Ruled surfaces can also be used in architecture. Doubly ruled surfaces are the inspiration for curved hyperboloid structures that can be built with a lattice work of straight elements. For example, saddle roofs can be given as an example of hyperbolic paraboloids. The shapes of cooling towers of powerstations are the examples of hyperboloid of one sheet. Also, ruled surfaces can be used for modeling spherical vaults, spiral staircases and splayed vaults. Many building materials can be considered as straightlines (e.g. woodstick) and can be benefited for creating ruled surfaces in architectural practices (Orbay, Kasap, \& Aydemir, 2009; Güngör \& Tosun, 2010).

As we said before, a differentiable curve on the dual unit sphere depending on a real parameter corresponds to a ruled surface in $R^{3}$, through using our equations, we can apply the E.study map of the spherical conics and this will be useful in the stated areas.

\section{References}

Altunkaya, B., Yaylı, Y., Hacısalihoğlu, H. H., \& Arslan, F. (2011). Equations of the spherical conics. Electronic journal of Mathematics and Technology, 5(3), 330-341.

Guggenheimer, H. W. (1977). Differential Geometry. New York: Dover Publications.

Güngör, M. A., \& Tosun, M. (2010). A Study on Dual Mannheim Partner Curves. International Mathematical Forum, 5(47), 2319-2330.

Hacısalihoğlu, H. H. (1972). On the pitch of a closed ruled surface. Mechanism and Machine Theory, 7, 291-305. http://dx.doi.org/10.1016/0094-114X(72)90039-0

Hacisalihoğlu, H. H. (1977). Study map of a circle. Journal of the Faculty of Science of Karadeniz Technical University, 1(7), 69-80. 
Kazaz, M., Özdemir, A., \& Uğurlu, H. H. (2008). Elliptic motion on dual hyperbolic unit sphere. Mechanism and Machine Theory, 44, 1450-1460. http://dx.doi.org/10.1016/j.mechmachtheory.2008.11.006

Körpınar, T., \& Turhan, E. (2010). On characterization dual spacelike biharmonic curves with spacelike principal normal according to dual Bishop frames inthe dual Lorentzian space $D_{1}^{3}$. Revista Notas de Mathematica, 6(2), No. 298, pp. 63-71.

Namikawa, Y. (1959). On Spherical Hyperbolas. (Japanese) Sugaku, 11.

Orbay, K., Kasap, E., \& Aydemir, I. (2009). Mannheim Offsets of Ruled Surfaces. Mathematical Problems in Engineering. http://dx.doi.org/10.1155/2009/160917

Study, E. (1903). Geometrie der Dynamen, Leibzig.

Veldkamp, G. R. (1976). On the use of dual numbers, vectors and matrices in instantaneous spatial kinematics. Mechanism and Machine Theory, 11. http://dx.doi.org/10.1016/0094-114X(76)90006-9

Wu, C. Y. (1995). Arbitrary Surface Flank Milling of Fan, Compressor, and Impeller Blades. J. Eng. Gas Turbines Power, 117(3), 534-539. http://dx.doi.org/10.1115/1.2814127

\section{Copyrights}

Copyright for this article is retained by the author(s), with first publication rights granted to the journal.

This is an open-access article distributed under the terms and conditions of the Creative Commons Attribution license (http://creativecommons.org/licenses/by/3.0/). 